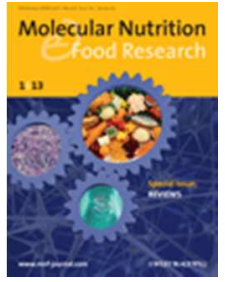

\title{
Determination of oleanolic acid in human plasma and its association with olive oil intake in healthy Spanish adults within the EPIC-Spain cohort study.
}

\begin{tabular}{|c|c|}
\hline Journal: & Molecular Nutrition and Food Research \\
\hline Manuscript ID & mnfr.201600927.R1 \\
\hline Wiley - Manuscript type: & Research Article \\
\hline Date Submitted by the Author: & $\mathrm{n} / \mathrm{a}$ \\
\hline Complete List of Authors: & $\begin{array}{l}\text { Buckland, Genevieve; Institut Catala d' Oncologia, Unit of Nutrition and } \\
\text { Cancer, Cancer Epidemiology Research Programme } \\
\text { Pastor, Antoni; IMIM (Hospital del Mar Medical Research Institute), , } \\
\text { Integrative Pharmacology and Systems Neuroscience Research Group, } \\
\text { Neurosciences Research Program; CIBER Fisiopatologia de la Obesidad y la } \\
\text { Nutrición (CIBERobn) } \\
\text { Lujan-Barroso, Leila; Institut Catala d' Oncologia, Unit of Nutrition and } \\
\text { Cancer, Cancer Epidemiology Research Programme; University of } \\
\text { Barcelona, Department of Nursing of Public Health, Mental Health and } \\
\text { Maternity and Child Health School of Nursing, } \\
\text { Gonzalez, Carlos; Institut Catala d' Oncologia, Unit of Nutrition and } \\
\text { Cancer, Cancer Epidemiology Research Programme } \\
\text { Travier, Noemie; Institut Catala d' Oncologia, Unit of Nutrition and Cancer, } \\
\text { Cancer Epidemiology Research Programme } \\
\text { Amiano, Pilar; BioDonostia Research Institute, Public Health Division of } \\
\text { Gipuzkoa; CIBER Epidemiology and Public Health (CIBERESP) } \\
\text { Huerta, Jose Maria; Murcia Regional Health Council, IMIB-Arrixaca, } \\
\text { Department of Epidemiology; CIBER Epidemiology and Public Health } \\
\text { (CIBERESP) } \\
\text { Agudo, A; Catalan Institute of Oncology, Epidemiology } \\
\text { Navarro, Carmen; Murcia Regional Health Council, IMIB-Arrixaca, } \\
\text { Department of Epidemiology; CIBER Epidemiology and Public Health } \\
\text { (CIBERESP) } \\
\text { Chirlaque, María Dolores; Murcia Regional Health Council, IMIB-Arrixaca, } \\
\text { Department of Epidemiology; CIBER Epidemiology and Public Health } \\
\text { (CIBERESP) } \\
\text { Sanchez, María José; Andalusian School of Public Health ; CIBER } \\
\text { Epidemiology and Public Health (CIBERESP) } \\
\text { Rodríguez Barranco, Miguel ; CIBER Epidemiology and Public Health } \\
\text { (CIBERESP) } \\
\text { Barricarte, Aurelio; Navarra Public Health Institute; CIBER Epidemiology } \\
\text { and Public Health (CIBERESP) } \\
\text { Ardanaz, Eva; Navarra Public Health Institute; CIBER Epidemiology and }\end{array}$ \\
\hline
\end{tabular}


2

3

4

5

6

7

8

9

\begin{tabular}{|l|l|}
\hline & $\begin{array}{l}\text { Public Health (CIBERESP) } \\
\text { Dorronsoro, Miren; BioDonostia Research Institute, Public Health Division } \\
\text { of Gipuzkoa; CIBER Epidemiology and Public Health (CIBERESP) } \\
\text { Amaia Úrsula, Molinuevo; CIBER Epidemiology and Public Health } \\
\text { (CIBERESP) } \\
\text { Quirós, J. Ramón; Public Health and Health Planning Directorate } \\
\text { de la Torre, Rafael; IMIM (Hospital del Mar Medical Research Institute), , } \\
\text { Integrative Pharmacology and Systems Neuroscience Research Group, } \\
\text { Neurosciences Research Program; CIBER Epidemiology and Public Health } \\
\text { (CIBERESP) }\end{array}$ \\
\hline Keywords: & $\begin{array}{l}\text { Olive oil, Oleanolic acid, Biomarker, Triterpenic compounds, EPIC-Spain } \\
\text { cohort }\end{array}$ \\
\hline
\end{tabular}

SCHOLARONE ${ }^{\text {' }}$

Manuscripts 


\title{
Determination of oleanolic acid in human plasma and its association with olive oil intake in healthy Spanish adults within the EPIC-Spain cohort study.
}

\author{
Genevieve Buckland $^{1}$, Antoni Pastor ${ }^{2,3}$, Leila Lujan-Barroso ${ }^{1,4}$, Carlos Alberto Gonzalez ${ }^{1}$, Noemie \\ Travier $^{1}$, Pilar Amiano ${ }^{5,6}$, José María Huerta ${ }^{5,7}$, Antonio Agudo ${ }^{1}$, Carmen Navarro ${ }^{5,7,8}$, María Dolores \\ Chirlaque $^{5,7,8}$, Maria-José Sánchez ${ }^{5,9}$, Miguel Rodríguez-Barranco ${ }^{9}$, Aurelio Barricarte ${ }^{5,10,11}$, Eva \\ Ardanaz $^{5,10,11}$, Miren Dorronsoro ${ }^{5,6}$, Amaia Molinuevo $^{5}$, J. Ramón Quirós ${ }^{12}$, Rafael de la Torre ${ }^{2,3,13}$
}

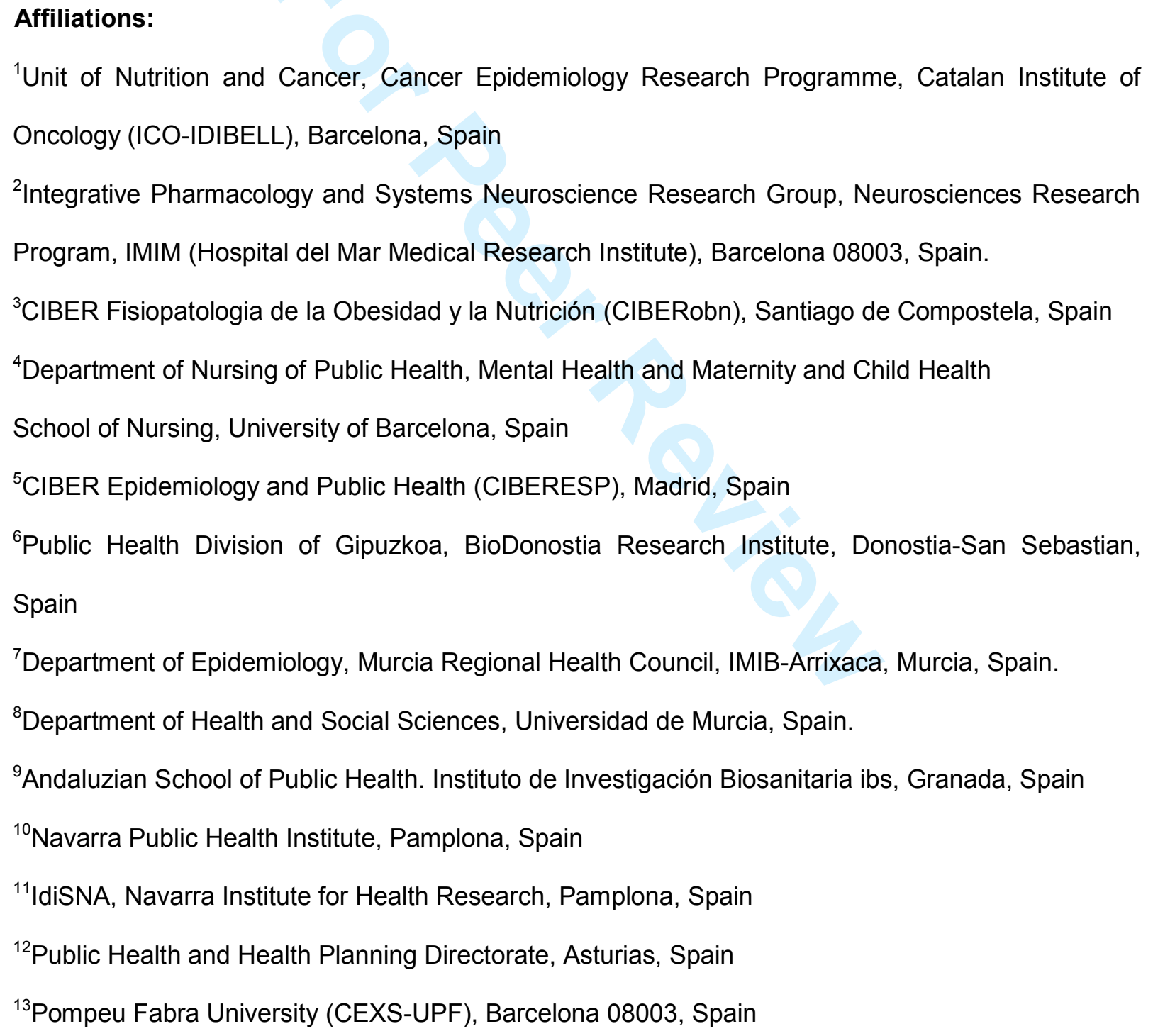




\author{
Corresponding Author: \\ Genevieve Buckland \\ Unit of Nutrition and Cancer \\ Cancer Epidemiology Research Programme \\ Catalan Institute of Oncology (ICO) \\ Avda Gran Via 199-203 \\ Barcelona, 08907, Spain \\ phone: 34/932607401 ext 3209 \\ fax: +34 932607787 \\ e-mail: gbuckland@iconcologia.net
}

\begin{abstract}
Abbreviations:
OA: Oleanolic Acid

EPIC: European Prospective Investigation into Cancer and Nutrition cohort study

SD: Standard Deviation
\end{abstract}

Key Words: Oleanolic acid, olive oil, biomarker, EPIC cohort study 


\begin{abstract}
Scope: Oleanolic acid (OA) is an important triterpenic compound found in olive oil, however little is known about its concentrations in human plasma. We aimed to determine plasma OA levels in a healthy Spanish population and compare them with estimates of dietary olive oil intake.
\end{abstract}

Methods and Results: The final study sample included 141 individuals randomly selected from the European Prospective Investigation into Cancer and Nutrition Spanish cohort. Dietary olive oil intake was estimated using validated dietary history questionnaires. OA concentrations were determined in plasma (from the participants' stored blood samples) using a high-performance liquid chromatography-mass spectrometry (HPLC-MS) method. Correlation coefficients between $\mathrm{OA}$ and olive oil intake were calculated, adjusting for centre, sex, age consumption of olives, apples, grapes and red wine and fasting state. The mean $\mathrm{OA}$ concentration in olive oil non-consumers was $0.72 \mathrm{ng} / \mathrm{ml}(\mathrm{SD} 0.82)$, while in the high olive oil intake group it was $1.32 \mathrm{ng} / \mathrm{ml}$ (SD 1.14). The fully adjusted partial Spearman correlations coefficients reached 0.36 ( $p$-value<0.001) overall, varying minimally by sex and fasting state.

Conclusion: This is the first study providing steady state concentrations of triterpenes in humans. The results show that there was low-to-moderate correlation between OA concentrations and olive oil intake in this population. 


\section{Introduction}

Oleanolic acid (OA) is a bioactive pentacyclic triterpenoid (3 $\beta$-hydroxyolean-12-en-28-oicacid, Figure 1) that exists widely in plants and medicinal herbs $(1,2)$. In particular, OA is one of the main triterpenic compounds, along with maslinic acid, found in the leaves from olive trees (Olea europaea L.) as well as in the skin of the olive fruit (1). Triterpenes are synthesized as a secondary plant metabolite, and OA acts as a defence barrier protecting olive leaves and fruit from pathogens such as insects, fungal attack and microorganisms (2).

One of the main dietary sources of this bitter astringent compound in Mediterranean countries is from consumption of table olives and olive oil, both of which form an integral part of the traditional dietary pattern. The concentration of this micro-constituent is in general higher in the olive fruit than in olive oil (1), and also varies depending on the quality of the oil, since the hydrolytic processes used to extract the oil from the fruit facilitates the release of the triterpenes from the skin (3). In extra virgin olive oil (acidity under 1\%) OA concentrations are on average $50 \mathrm{mg} / \mathrm{kg}$, in virgin olive oil OA concentrations are generally over $200 \mathrm{mg} / \mathrm{kg}$, while olive pomace oils can have very high amounts of OA, ranging from $2000-8000 \mathrm{mg} / \mathrm{kg}$ (3). Other relevant dietary sources within Mediterranean populations are fruit, including olives, grapes and apples (4-6) and OA is particularly concentrated in the fruit peel (6).

OA is becoming increasingly recognised for its wide range of important biological activities which are reported to provide beneficial health effects, including anti-inflammatory, antidiabetic, anti-tumoral, hepato-protective, cardioprotective and neuroprotective effects $(1,2,7-9)$. However, these beneficial properties have mainly been studied in experimental research; in vitro or in animal models (1,10-12). In addition, the extent by which OA 
contributes to the protective effect seen from consuming olive oil on risk of major chronic diseases(13) is also unclear; so far olive oil's benefits have mainly been ascribed to its high content of monounsaturated fatty acids, in particular oleic acid, and also to other minor ( 1$2 \%$ of oil weight) but highly bioactive hydrophilic compounds such as phenol compounds (14-16).

OA may also be a potential biomarker of olive oil intake and could provide useful information for studying nutritional epidemiology. This is particularly relevant because of the measurement error in estimating olive oil consumption in observational studies. Information on intake is usually provided by self-reported dietary assessments such as dietary histories, food frequency questionnaires or 24-hour dietary recalls. All of these methods are subject to measurement error, to a lesser or greater extent depending on the method. Estimating olive oil consumption is particularly challenging, because as well as being added to salads and vegetable dishes during mealtimes it is also used habitually in cooking, so precise quantity estimates can be complicated. Therefore, identifying specific nutritional biomarkers that reflect olive oil intake is important as it could then be applied in the study of dietdisease associations. Hydroxytyrosol and tyrosol are well recognised biomarkers of olive oil intake and a dose-response relationship has been reported $(17,18)$. However, these phenolic compounds have a relatively short half-life (2.4 hours for hydroxytyrosol (18)), and therefore are unfortunately not suitable for many prospective epidemiological studies where blood samples are often taken in the morning in a fasting state. In addition, wine and ethanol consumption are confounding factors when using hydroxytyrosol as biomarker of olive oil consumption (19). In contrast, OA has been reported to have a longer half-life, of 8.7 hours, and approximately $3 \mathrm{ng} / \mathrm{ml}$ of $\mathrm{OA}$ was found in plasma 15 hours after administration of an oral dose of $40 \mathrm{mg}$ of OA (20). 
Despite its importance, little is known about the concentrations of OA in human subjects, in particular in Mediterranean populations with a regular use of olive oil. Whether plasma OA can be used as a biomarker for olive oil consumption is also not clear. Therefore, the objective of this study was to measure levels of OA in human plasma samples taken from healthy volunteers within the European Prospective Investigation into Cancer and Nutrition (EPIC) Spain cohort study and compare plasma OA concentrations with dietary estimates of olive oil intake from baseline diet history questionnaires.

\section{Materials and Methods}

Study sample

This study is based on participants from the EPIC-Spain cohort $(21,22)$, which includes 41,438 volunteers aged 29-69 years old, recruited between 1992 and 1996 from three regions in the north (Asturias, Gipuzkoa and Navarra) and two regions in the south (Granada and Murcia). The participants were from different social and educational levels and recruited mostly from blood donors. At recruitment subjects gave their informed consent and the study was approved by the Medical Ethical Committee of Bellvitge Hospital. A random subset $(\sim 7 \%)$ of individuals from the EPIC-Spain cohort took part in a second dietary measurement for calibration purposes (23) and also had plasma samples available $(n=2,763)$. Of these, 317 participants had been diagnosed with incident cardiovascular disease, cancer or diabetes, or were dead at last follow-up date and were excluded, leaving 2,446 participants eligible for inclusion in our study (the sampling population). 
Information on usual diet and lifestyle factors and anthropometry were obtained at recruitment. A computerized version of a validated dietary history questionnaire was used across all EPIC-Spain centres to collect information on usual food intake over the previous 12 months $(24,25)$. The questionnaire was open but was structured by meals and included a list of approximately 600 common foods and recipes from each region. The frequency and amount of food consumed at least twice a month was recorded, taking seasonal variability into account. The portion of each food (grams/day) consumed was quantified by using household measures, standard units, and a collection of 35 sets of photographs of simple foods, mixed foods, and drinks. Oil added to salads and cooked foods was measured by using standard household measures (24). Total energy (kilocalories/day) and daily nutrient intake were estimated by using a food composition table (26). A 30mL blood sample was also obtained at recruitment (60\% of all study participants were in fasting state), and divided into $0.5 \mathrm{~mL}$ aliquots of plasma, serum, red blood cells, and buffy coat, and stored in liquid nitrogen at minus $196^{\circ} \mathrm{C}$.

\section{Sample size calculations}

We estimated that we would need 47 subjects in each of the three groups (non-consumers and low and high consumers of olive oil), to be able to detect a minimum difference of $0.5 \mathrm{ng} / \mathrm{ml}$ of $\mathrm{OA}$ in human plasma, calculated applying a type I error $(\alpha=0.05)$ and with a power of $80 \%$, and based on estimations of OA concentrations and coefficient of variation from the previously published pharmacokinetics study (20). Therefore, a minimum of 141 subjects in total would be required, which was rounded up to 150 to take into account sample losses (either due to sample extraction or evaluation). The random selection of 150 individuals was done by first assigning a random number to the 2,446 subjects in sampling 
population, using STATA statistical software (version 10; StataCorp LP.). The subjects were then divided into three groups, according to their olive oil intake from the dietary history questionnaire. The groups were i) non-consumers of olive oil ( $0 \mathrm{~g} / \mathrm{day})$, ii) low consumers of olive oil (subjects within the lowest tertile of olive oil intake within consumers) and iii) high consumers of olive oil (subjects within the highest tertile of olive oil intake within consumers). Olive oil consumers include subjects reporting the use of exclusively ordinary olive oil (a blend of refined olive oil and virgin olive oils), exclusively virgin olive oil or a combination of ordinary and virgin olive oil (no information on extra-virgin olive oil was available). A total of 50 subjects were then selected, using the random number previously assigned to all subjects, from each of these 3 groups in order to identify the 150 subjects needed for our study. The randomisation programme was designed to as evenly as possible represent equal numbers of men and women and subjects from each of the 5 EPIC-Spain centres.

\section{Determination of $O A$}

The method of analysis of oleanolic acid in plasma was based largely on a validated method previously described (20). Calibration plasma samples were prepared with a pool of oleanolic-free plasma obtained from five healthy volunteers that followed a one week exclusion of olive oil and olive-derived products from their diet. Calibration curves were freshly prepared in duplicate for each batch of analysis by spiking oleanolic acid stock standard solution (HWI ANALYTIC GMBH, Ruelzheim, Germany) to $1 \mathrm{~mL}$ plasma. Aliquots of $1 \mathrm{~mL}$ of plasma from the study subjects, calibration samples and quality control samples were transferred to $12 \mathrm{~mL}$ glass tubes, spiked with $25 \mu \mathrm{L}$ of internal standard oleanolic acidd3 (Toronto Research Chemicals, North York, Canada) solution (1.0 $\mu \mathrm{g} / \mathrm{mL})$, diluted with $1 \mathrm{~mL}$ of $1 \%$ sodium chloride solution (water) and extracted with $6 \mathrm{~mL}$ ethyl acetate by placing 
the tubes in a rocking mixer over $20 \mathrm{~min}$. After centrifugation (5min, 1700g), the organic layers were separated into clean $12 \mathrm{~mL}$ glass tubes and placed in a water bath $\left(29^{\circ} \mathrm{C}\right)$ under a stream of nitrogen (40-50 min). The dry organic extracts were reconstituted in $100 \mu \mathrm{L}$ of a mixture methanol: $35 \mathrm{mM}$ ammonium acetate in water $(85: 15, \mathrm{v} / \mathrm{v})$ and transferred to HPLC vials with microvials. Twenty $\mu \mathrm{L}$ were injected into the LC/MS-MS system. An Agilent 6410 triple quadrupole (Agilent Technologies, Wilmington, DE) coupled to a chromatographic system consisting in a 1200 series binary pump, a column oven $\left(40^{\circ} \mathrm{C}\right)$ and a cooled autosampler $\left(4^{\circ} \mathrm{C}\right)$ were used. Chromatographic separation was carried out by gradient chromatography with a Waters C18-CSH column $(3.1$ x $100 \mathrm{~mm}, 1.8 \mu \mathrm{m}$ particle size $)$ maintained at $40^{\circ} \mathrm{C}$ with a mobile phase flow rate of $0.35 \mathrm{~mL} / \mathrm{min}$. The composition of the mobile phase was: A: methanol: $35 \mathrm{mM}$ ammonium acetate in water $(85: 15, \mathrm{v} / \mathrm{v})$, and $\mathrm{B}$ : methanol. The initial conditions were $20 \% \mathrm{~B}$. The gradient was first increased linearly to $100 \%$ B over 6 minutes, maintained at $100 \%$ B for 1 minute, to return to initial conditions for a further 5 minutes with a total run time of 12 minutes. The electrospray ion source was set on the negative ionization mode. The mass spectrometry detection was done by single reaction monitoring $(\mathrm{SRM})$ of the following transitions: $\mathrm{m} / \mathrm{z} 455.2>455.2$ (oleanolic acid), and $\mathrm{m} / \mathrm{z} 458.3>458.3$ (oleanolic acid-d3). The collision energies of oleanolic acid and oleanolic acid-d3 were $20 \mathrm{eV}$ in both cases. Quantification was done by interpolation into the calibration curve of the ratio of the peak area of the analyte vs the peak area of the internal standard. The limit of detection of the method was $0.3 \mathrm{ng} / \mathrm{mL}$ and accuracy (error) and precision were $<15 \%$. Figure 2 shows an LC/MS-MS chromatogram of the analysis of a human plasma sample.

\section{Statistical analysis}

From the initial 150 study participants selected, one plasma sample could not be retrieved and for three participants no chromatography signal was obtained, leaving a total of 146 
participants with complete information on OA concentrations. Out of these 146 participants, we excluded 5 participants who were identified as having extremely high OA concentrations, defined as values above $5 \mathrm{ng} / \mathrm{mL}$, since these levels have only been reported in human plasma samples after taking high doses of OA supplements. This left a total of 141 participants for the final study analysis. Olive oil intake data from the diet history questionnaire and concentrations of $\mathrm{OA}$ in plasma were calculated as means and standard deviation (SD) and medians and inter-quartile range, stratified by sex and by the 5 EPICSpain centres, and according to olive oil intake group (non-consumers, all consumers, low intake and high intake of olive oil). Further stratification of low and high intake groups into type of olive oil consumed was not feasible due to the limited number of subjects in each of these sub-groups. The OA values which were below the limit of detection $(0.3 \mathrm{ng} / \mathrm{ml})$ were given a value of $0.15 \mathrm{ng} / \mathrm{ml}$ (in total 35 values were adjusted; 16 values from olive oil nonconsumers, 14 values from low olive oil consumers and 5 values from high olive oil consumers). Normality of data on olive oil intake and OA was tested using Shapiro-Wilk test. The differences between the mean OA concentration in different olive oil intake groups was tested using non-parametric Wilcoxon rank-sum test, since both OA and olive oil intake were non-normally distributed.

We evaluated the correlation between plasma OA concentrations and olive oil intake using Spearman non-parametric tests, restricting analyses to the olive oil consumers subgroup $(n=96)$. Spearman partial correlation coefficients were calculated adjusting for centre, age and sex in a partially adjusted model and in a fully adjusted model additionally adjusting for potential dietary sources of OA such as red wine and total fruit intake (which includes apples (the most consumed fruit in the EPIC-Spain cohort), grapes and olives and other fruit which may contain OA) as well as fasting state. Results are also presented for sexspecific models and models stratified by fasting state ( $n=49$ fasting vs $n=47$ non-fasting). 
Data were analysed using Stata version 10 (Statacorp, College Station, TX) and SAS software.

\section{Results}

A description of the baseline characteristics of the 141 participants ( $47 \%$ male) included in the analysis, according to olive oil intake, is presented in Table 1 . There were a total of 45 non-consumers of olive oil, 49 subjects with a low olive oil intake and 47 subjects with a high olive oil intake. Approximately a quarter of the sample (27\%) had a secondary school education or above, a large proportion were overweight or obese (85\%), and roughly half of the sample were never smokers (55\%).

Table 2 presents the mean olive oil intake and OA concentration overall and by sex and test of difference of mean OA concentration between different olive oil intake groups. Since there were very few subjects in certain EPIC centres and olive oil intake groups (shown in Table 1), the olive oil and OA concentrations are not presented by centre. The mean olive oil intake was $24.5 \mathrm{~g} /$ day (SD 17.2) in olive oil consumers, $9.2 \mathrm{~g} / \mathrm{d}$ (SD 4.5) in the low olive oil intake group and $40.4 \mathrm{~g} /$ day (SD 9.2) in the high olive oil intake group. Olive oil intake in the low category ranged from $0.86 \mathrm{~g} /$ day to $16.51 \mathrm{~g} /$ day, while in the high olive oil category intake ranged from $28.56 \mathrm{~g} /$ day to $70.43 \mathrm{~g} /$ day (data not shown). Although the mean olive oil intake was higher in men than women in all of the olive oil intake groups, these sex differences were not statistically significant ( $p$-value 0.515 for all olive oil consumers, $p$ value 0.172 for low olive oil intake and $p$-value 0.429 for high olive oil intake group).

The mean OA concentration in non-consumers of olive oil was $0.72 \mathrm{ng} / \mathrm{ml}$ (SD 0.82 ), in the low olive oil intake group it was $0.95 \mathrm{ng} / \mathrm{ml}$ (SD 1.03) while in the high olive oil intake group it increased to $1.32 \mathrm{ng} / \mathrm{ml}$ (SD 1.14). The mean OA concentration was in general higher in men than women, but these differences were not statistically significant ( $p$-values 0.505 for 
non-consumers, p-value 0.658 for all consumers, $p$-value 0.321 for low olive oil intake group and p-value 0.992 for high olive oil intake group). There was a statistically significant difference in the mean OA concentration between the group of olive oil non-consumers and all consumers $(p=0.012)$, non-consumers and the high olive oil intake group $(p=0.002)$, and a borderline significant difference between the low and high olive oil group $(p=0.052)$. However, there was no significant difference in OA between olive oil non-consumers and low olive oil intake group ( $p=0.213)$. In additional analyses (data not tabulated) there was no statistically significant difference $(p=0.359)$ between the mean OA concentration in fasting blood samples $(0.89 \mathrm{ng} / \mathrm{ml}(\mathrm{n}=85))$ and non-fasting samples $(1.20 \mathrm{ng} / \mathrm{ml}(\mathrm{n}=56))$.

Table 3 shows the correlation coefficients for OA concentration and olive oil intake among olive oil consumers, for crude and adjusted models. In the crude model the overall correlation was 0.18 ( $p$-value=0.072), which increased to 0.37 (p-value<0.001) in the partially adjusted model. After additional adjustment for total fruit intake, red wine consumption and fasting state the correlations were attenuated very slightly $(r=0.36$; $p$ value $<0.001)$. The correlations between men and women in the adjusted models showed minimal differences. In fully adjusted models stratified by the participants' fasting state the correlations were also similar between those in a fasting state $(r=0.40 ; p$-value 0.009$)$ and those in a non-fasting state $(r=0.35 ; p$-value 0.03) (data not tabulated).

\section{Discussion}

This is the first study providing steady state concentrations of triterpenes in humans with a mean concentration ranging from $0.72 \mathrm{ng} / \mathrm{mL}$ in non-olive oil consumers to $1.32 \mathrm{ng} / \mathrm{mL}$ among high olive oil consumers. The estimated mean consumption of olive oil in our study population ( $24.5 \mathrm{~g} / \mathrm{day})$ is similar to the Spanish population participating in the SUN cohort 
study (24.9 g/day), which also reported higher intake levels in men compared to women (27). The correlation between OA concentrations and dietary olive oil intake in this population was low-to-moderate ( $r=0.36$ overall). However, these values are comparable to correlations between other biomarkers and nutrient intakes obtained from dietary questionnaires in the framework of the EPIC study, with few correlation coefficients over $0.50(28,29)$.

The mean OA concentration in our study $(1.13 \mathrm{ng} / \mathrm{ml}$ in olive oil consumers) was lower than expected, although there is very limited data in this area and none to date in the general population in a non-experimental setting. Furthermore, a direct comparison with data from the pharmacokinetics study by Song et al. (20) should be made with caution, since the 18 healthy volunteers were given a $40 \mathrm{mg}$ dose of $\mathrm{OA}$, a much higher concentration than would be found in the habitual use of olive oil (3), which our participants were exposed to.

Despite the relatively low OA concentrations compared to other minor compounds found in olive oil (30), other triterpenes like maslinic acid may be complementary in terms of biological activity, although they have yet to be determined. The health benefits of triterpenes in humans need to be re-evaluated on the light of body concentrations of these compounds. Nevertheless, concentrations of $\mathrm{OA}$ in plasma are able to differentiate populations consuming olive oil and populations with high olive oil consumption from nonconsumers. However, they cannot differentiate the low olive oil consumers from the nonconsumers. This is because there is a background concentration of OA in plasma despite not consuming olive oil. Other sources of OA, as stated earlier, could be the peels of fruits common in the Mediterranean diet, particularly apples and grapes (5). Wine is another source of $\mathrm{OA}$, although the transfer of triterpenoids from grape peels to wine may not be 
particularly relevant and a rough estimate particularly from red wines (which have a higher concentration) is that triterpene concentrations are about 20 times lower than in olive oil per $\mathrm{kg}$ of product (31). However, this is partially counterbalanced by the volumes in $\mathrm{mL}$ of both products consumed daily. Brandies along with red wine also contain triterpenes (not specifically $\mathrm{OA}$ ), in this case in larger concentrations, but their incorporation in the beverage is largely related to their transfer from oak heartwood barrels in the process of aging. Despite these other dietary sources of OA, adjusting for the habitual consumption of total fruit intake and red wine only fractionally attenuated the correlation coefficients between plasma OA concentrations and olive oil intake. Although we did not have information on consumption of fruit and red wine over the 24 hours previous to the blood draw, when the analyses were restricted to those in a fasting state (to reduce the influence of differences between participants in recent consumption of these other dietary sources of OA as well as recent olive oil intake) the correlations were very similar.

Previous biomarkers of olive oil ingestion, such as tyrosol and hydroxytyrosol in urine, have proven to be efficient in differentiating nutritional interventions with olive oils with varying amounts of phenolic compounds $(17,18)$, but the cross-contamination with wine consumption and changes in dopamine oxidative metabolism after ethanol ingestion limit their generalization in future studies (30). Studies performed with these biomarkers were exclusively performed in urine. The analytical methodology for the analysis of hydroxytyrosol in plasma was not available until recently (32). Therefore correlation studies like the ones performed in the present study have not been yet performed.

The study has certain strengths and limitations. It is the first study of this type to assess steady state OA and olive oil intake correlation in a healthy free-living population. This has 
been possible by applying a highly accurate and sensitive method allowing determining OA concentrations in plasma in a Mediterranean population with regular use of olive oil. The effects of long-term sample storage (EPIC blood samples collected over 10 years ago) on OA concentrations are unknown. In a previous study OA concentrations were stable in plasma at $-20^{\circ} \mathrm{C}$ for at least 30 days (20). Similar results were observed during laboratory tests by the authors of the present report (data not published). In addition, plasma samples subjected to at least three cycles of thaw and freezing did not show alterations in plasma concentrations. Therefore, globally $\mathrm{OA}$ seems to be stable in plasma stored at $-20^{\circ} \mathrm{C}$.

Another issue to be considered is measurement errors in estimation of dietary olive oil intake, however in EPIC we used dietary history questionnaires which are shown to have less errors than food frequency questionnaires(29), and measurement errors are likely to be restricted to estimates within olive oil consumers but not affect the non-consumers group. In addition, dietary history questionnaires that were used in our study capture habitual consumption of olive oil but do not provide detailed information on consumption over the previous 24 hours (which was not available in our study). Since the half life of OA is approximately eight hours and just over half the subjects were in a fasting state when blood samples were taken this could have been relevant, and may explain the lower than expected OA concentrations. Indeed in the pharmacokinetics study (20), 20 hours after the $40 \mathrm{mg}$ oral dose of OA, plasma OA concentrations had dropped to approximately $2 \mathrm{ng} / \mathrm{ml}$ (from a peak of approximately $9 \mathrm{ng} / \mathrm{ml}$ after 5 hours). However, after adjusting for fasting state in correlation analyses there were only marginal changes in the correlation results.

Another point to consider is that the power calculations were based on an anticipated minimum difference in mean plasma $O A$ concentration of $0.5 \mathrm{ng} / \mathrm{ml}$ for participants in 
different olive oil groups, which was not always reached (i.e. for non-consumers and low olive oil intake groups there was a mean difference of $0.23 \mathrm{ng} / \mathrm{ml}$ ). This could have lead to reduced power $(<80 \%)$ to detect statistical differences between these groups. However, the difference between non-consumers and high olive oil consumers was actually more $(0.6 \mathrm{ng} / \mathrm{ml})$, and so would have been adequately powered. Although OA concentrations vary depending on the quality of the olive oil, the study sample size was not calculated in order to analyze differences in OA concentrations between in exclusive consumers of ordinary $(n=49)$ or virgin $(n=34)$ olive oil (only 13 subjects consumed both types). Despite this we found significant or borderline significant differences in OA concentrations between most of the olive oil groups (overall). The lack of information on consumption of extra-virgin olive oil may be less relevant though since OA concentrations are reported to be much lower in this type of olive oil (3).

OA plasma concentrations may also be confounded by other foods containing OA that were not controlled for, but their contribution should be minor considering concentration levels reported in other plant sources (6). Confounding or interactions with other unknown factors conditioning blood concentration levels cannot be ruled out though due to lack of data in this area. Finally, the non-significant results observed in several analyses in men and women assessing the difference between the mean OA concentrations in different olive oil groups, may have been due to smaller numbers of individuals in these sub-analyses, resulting in insufficient statistical power.

\section{Concluding Remarks}


In this study OA concentrations in blood were able to differentiate between the groups of individuals with more extremes of olive oil consumption. However, due to various limiting factors outlined above OA's potential as a more sensitive dietary biomarker, distinguishing between smaller differences in olive oil intake, needs to be further clarified. Definitive studies to better determine OA and possibly other triterpenes such as maslinic acid as olive oil biomarkers should involve nutritional intervention studies with controlled doses of triterpenes (to rule out measurement error of dietary questionnaires), using olive oil of different qualities and should determine OA concentration over time in blood and urine.

Authors Contributions: AP carried out the laboratory analyses. GB, AP and RT jointly wrote the article. GB, LLB and NT carried out the statistical analysis. All authors were involved in the study design, interpretation of the results and discussion.

\section{Conflicts of Interest: None}

Acknowledgements: AGAUR, Generalitat de Catalunya (exp. 2014 SGR 726); The Health Research Funds (FIS Exp PI12/00335 and Exp PI15/00646) and (RD12/0036/0018); Spanish Regional Governments of Andalusia, Asturias, Basque Country, Murcia (No. 6236) and Navarra; the Catalan Institute of Oncology (ICO-IDIBELL); CIBER Epidemiología y Salud Pública (CIBERESP) 
Reference List

(1) Sanchez-Quesada C, Lopez-Biedma A, Warleta F, Campos M et al. Bioactive properties of the main triterpenes found in olives, virgin olive oil, and leaves of Olea europaea. J Agric Food Chem. 2013, 61, 12173-12182.

(2) Pollier J, Goossens A. Oleanolic acid. Phytochemistry. 2012, 77, 10-15.

(3) Perez-Camino MC, Cert A. Quantitative determination of hydroxy pentacyclic triterpene acids in vegetable oils. J Agric Food Chem. 1999, 47, 1558-1562.

(4) Kanellos PT, Kaliora AC, Gioxari A, Christopoulou GO et al. Absorption and bioavailability of antioxidant phytochemicals and increase of serum oxidation resistance in healthy subjects following supplementation with raisins. Plant Foods Hum Nutr. 2013, 68, 411-415.

(5) Pensec F, Paczkowski C, Grabarczyk M, Wozniak A et al. Changes in the triterpenoid content of cuticular waxes during fruit ripening of eight grape (Vitis vinifera) cultivars grown in the Upper Rhine Valley. J Agric Food Chem. 2014, 62, 7998-8007.

(6) Jager S, Trojan H, Kopp T, Laszczyk MN, Scheffler A. Pentacyclic triterpene distribution in various plants - rich sources for a new group of multi-potent plant extracts. Molecules. 2009, 14, 2016-2031.

(7) Zhang W, Men X, Lei P. Review on anti-tumor effect of triterpene acid compounds. J Cancer Res Ther. 2014, 10, S14-S19. 
(8) Camer D, Yu Y, Szabo A, Huang XF. The molecular mechanisms underpinning the therapeutic properties of oleanolic acid, its isomer and derivatives for type 2 diabetes and associated complications. Mol Nutr Food Res. 2014, 58, 1750-1759.

(9) Sanchez-Quesada C, Lopez-Biedma A, Gaforio JJ. Oleanolic Acid, a Compound Present in Grapes and Olives, Protects against Genotoxicity in Human Mammary Epithelial Cells. Molecules. 2015, 20, 13670-13688.

(10) Juan ME, Planas JM, Ruiz-Gutierrez V, Daniel H, Wenzel U. Antiproliferative and apoptosis-inducing effects of maslinic and oleanolic acids, two pentacyclic triterpenes from olives, on HT-29 colon cancer cells. Br J Nutr. 2008, 100, 36-43.

(11) Juan ME, Wenzel U, Ruiz-Gutierrez V, Daniel H, Planas JM. Olive fruit extracts inhibit proliferation and induce apoptosis in HT-29 human colon cancer cells. $J$ Nutr. 2006, 136, 2553-2557.

(12) Alqahtani A, Hamid K, Kam A, Wong KH et al. The pentacyclic triterpenoids in herbal medicines and their pharmacological activities in diabetes and diabetic complications. Curr Med Chem. 2013, 20, 908-931.

(13) Buckland G, Gonzalez CA. The role of olive oil in disease prevention: a focus on the recent epidemiological evidence from cohort studies and dietary intervention trials. Br J Nutr. 2015, 113, S94-S101.

(14) Lopez-Miranda J, Perez-Jimenez F, Ros E, De Caterina R et al. Olive oil and health: summary of the II international conference on olive oil and health consensus report, Jaen and Cordoba (Spain) 2008. Nutr Metab Cardiovasc Dis. 2010, 20, 284-294. 
(15) Covas MI, Nyyssonen K, Poulsen HE, Kaikkonen J et al. The effect of polyphenols in olive oil on heart disease risk factors: a randomized trial. Ann Intern Med. 2006, 145, 333-341.

(16) Silva S, Combet E, Figueira ME, Koeck T et al. New perspectives on bioactivity of olive oil: evidence from animal models, human interventions and the use of urinary proteomic biomarkers. Proc Nutr Soc. 2015, 74, 268-281.

(17) Miro-Casas E, Covas MI, Fito M, Farre-Albadalejo M et al. Tyrosol and hydroxytyrosol are absorbed from moderate and sustained doses of virgin olive oil in humans. Eur J Clin Nutr. 2003, 57, 186-190.

(18) Miro-Casas E, Covas MI, Farre M, Fito M et al. Hydroxytyrosol disposition in humans. Clin Chem. 2003, 49, 945-952.

(19) Schroder H, De La Torre R, Estruch R, Corella D et al. Alcohol consumption is associated with high concentrations of urinary hydroxytyrosol. Am J Clin Nutr. 2009, $90,1329-1335$.

(20) Song M, Hang TJ, Wang $Y$, Jiang $L$ et al. Determination of oleanolic acid in human plasma and study of its pharmacokinetics in Chinese healthy male volunteers by HPLC tandem mass spectrometry. J Pharm Biomed Anal. 2006, 40, 190-196.

(21) Riboli E, Hunt KJ, Slimani N, Ferrari P et al. European Prospective Investigation into Cancer and Nutrition (EPIC): study populations and data collection. Public Health Nutr. 2002, 5, 1113-1124.

(22) Gonzalez CA. The European Prospective Investigation into Cancer and Nutrition (EPIC). Public Health Nutr. 2006, 9, 124-126. 
(23) Slimani N, Kaaks R, Ferrari P, Casagrande C et al. European Prospective Investigation into Cancer and Nutrition (EPIC) calibration study: rationale, design and population characteristics. Public Health Nutr. 2002, 5, 1125-1145.

(24) González CA. Relative validity and reproducibility of a diet history questionnaire in Spain. I. Foods. Int J Epidemiol. 1997, 26, 91-99.

(25) González CA. Relative validity and reproducibility of a diet history questionnaire in Spain. II. Nutrients. Int J Epidemiol. 1997, 26, 100-109.

(26) Slimani N, Deharveng G, Unwin I, Southgate DA, Vignat J, Skeie G et al. The EPIC nutrient database project (ENDB): a first attempt to standardize nutrient databases across the 10 European countries participating in the EPIC study. Eur J Clin Nutr. $2007,61,1037-1056$.

(27) Fernandez-Jarne E, Martinez-Losa E, Prado-Santamaria M, Brugarolas-Brufau et al. Risk of first non-fatal myocardial infarction negatively associated with olive oil consumption: a case-control study in Spain. Int J Epidemiol. 2002, 31, 474-480.

(28) Kaaks R, Riboli E. Validation and calibration of dietary intake measurements in the EPIC project: Methodological considerations. Int J Epidemiol. 1997, 26, S15-S25.

(29) Kaaks R, Slimani N, Riboli E. Pilot phases studies on the accuracy of dietary intake measurements in the EPIC porject: overall evaluation of results. Int $J$ Epidemiol. 1997, 26, S26-S36.

(30) Rodriguez-Morato J, Boronat A, Kotronoulas A, Pujadas M et al. Metabolic disposition and biological significance of simple phenols of dietary origin: hydroxytyrosol and tyrosol. Drug Metab Rev. 2016, 48, 218-236 
(31) Arramon G, Saucier C, Tijou S, Glories Y. Estimation of Triterpenes in Wines, Spirits and Oak Heartwoods. LCGC North America; 2003.

(32) Pastor A, Rodriguez-Morato J, Olesti E, Pujadas M et al. Analysis of free hydroxytyrosol in human plasma following the administration of olive oil. $J$ Chromatogr A. 2016, 1437, 183-190. 
Table 1. The baseline characteristics of the study sample, according to olive oil intake based on dietary history questionnaire data.

\begin{tabular}{|c|c|c|c|c|}
\hline \multirow{2}{*}{ Sample characteristics } & \multirow{2}{*}{ Total } & \multicolumn{3}{|c|}{ Olive oil intake group } \\
\hline & & Non-consumers & Low intake & High intake \\
\hline Participants, n (\% male) & $141(47)$ & $45(53)$ & $49(33)$ & $47(55)$ \\
\hline \multicolumn{5}{|l|}{ Center, $\mathrm{n}$} \\
\hline Asturias & 28 & 11 & 12 & 5 \\
\hline Granada & 31 & 1 & 19 & 11 \\
\hline Murcia & 25 & 11 & 7 & 7 \\
\hline Navarra & 32 & 8 & 6 & 18 \\
\hline San Sebastián & 25 & 14 & 5 & 6 \\
\hline \multicolumn{5}{|l|}{ Educational level - secondary } \\
\hline school or above, $\mathrm{n}(\%)$ & $37(26)$ & $10(22)$ & $13(27)$ & $14(30)$ \\
\hline BMI - $\geq 25 \mathrm{~kg} / \mathrm{m}^{2}, \mathrm{n}(\%)$ & $123(87)$ & $40(89)$ & $42(85)$ & $41(87)$ \\
\hline \multicolumn{5}{|l|}{ Smoking status, $\mathrm{n}(\%)$} \\
\hline Never & $78(55)$ & $26(58)$ & $28(57)$ & $24(51)$ \\
\hline Former & $29(21)$ & $8(18)$ & $9(18)$ & $12(26)$ \\
\hline Current & $34(24)$ & $11(24)$ & $12(24)$ & $11(23)$ \\
\hline \multicolumn{5}{|l|}{ Physical activity level - } \\
\hline moderately active/active, $\mathrm{n}(\%)$ & $35(25)$ & $12(27)$ & $8(16)$ & $15(32)$ \\
\hline
\end{tabular}


Figure 1. Chemical structure of Oleanolic Acid
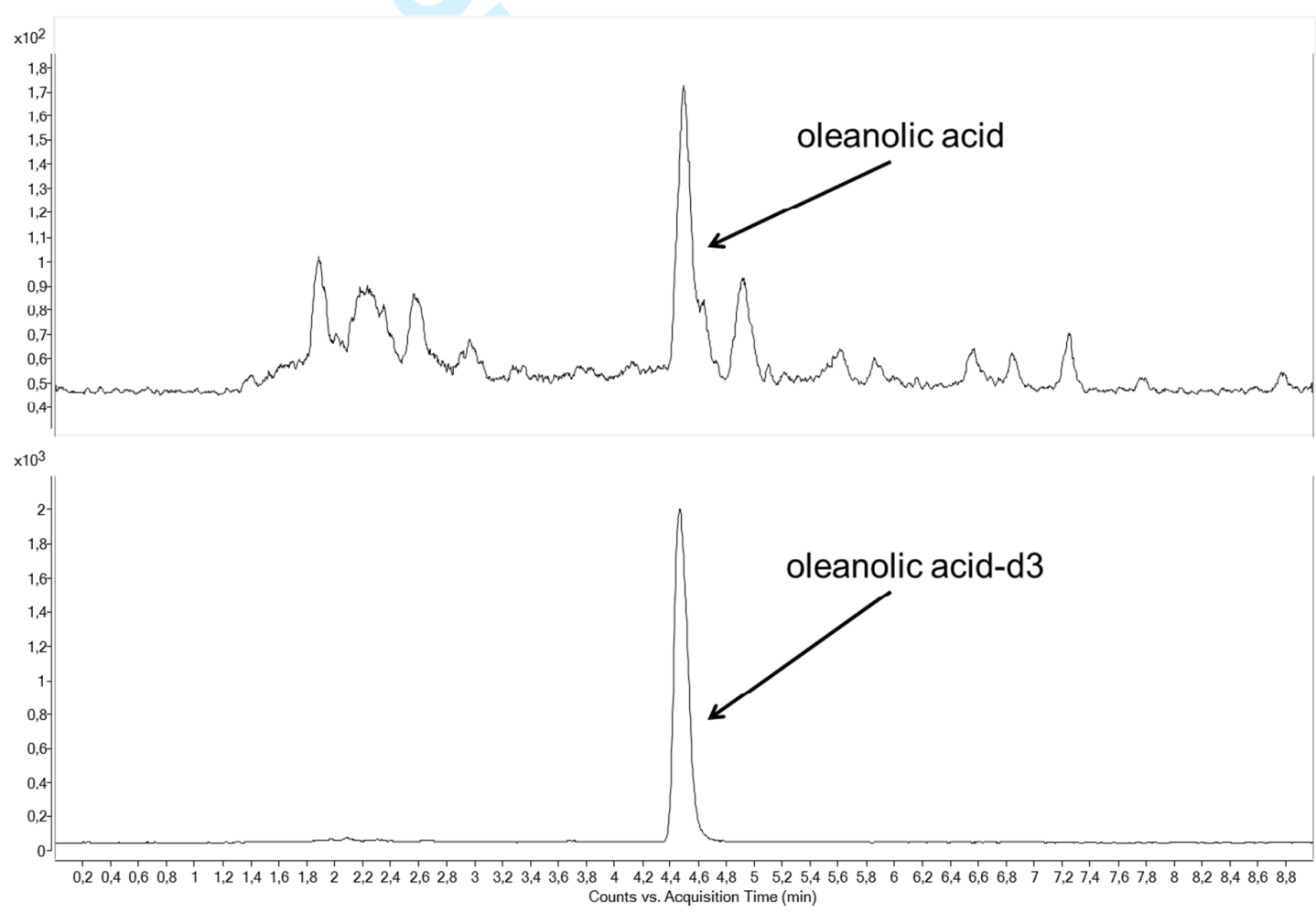

Figure 2. LC/MS-MS chromatogram of a human plasma sample with a calculated concentration of $1.73 \mathrm{ng} / \mathrm{mL}$ oleanolic acid showing the peak of oleanolic acid $(\mathrm{m} / \mathrm{z} 455>455)$ and the peak of the internal standard oleanolic acid-d3 (m/z $458>458)$ 
Table 2. Olive oil intake and oleanolic acid concentration overall and by sex, according to the olive oil intake groups based on the dietary questionnaire data.

\begin{tabular}{|c|c|c|c|c|c|c|}
\hline \multirow{2}{*}{$\begin{array}{l}\text { OA concentration and olive } \\
\text { according to intake group }\end{array}$} & \multicolumn{2}{|c|}{ Total $(n=141)$} & \multicolumn{2}{|c|}{ Male $(n=66)$} & \multicolumn{2}{|c|}{ Female $(n=75)$} \\
\hline & Mean (sd) & Median (p25-p75) & Mean (sd) & Median (p25-p75) & Mean (sd) & Median (p25-p75) \\
\hline Olive oil intake g/day & & 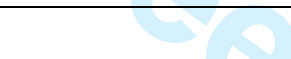 & & & & \\
\hline All olive oil consumers & $24.5(17.2)$ & $16.5(7.9-38.1)$ & $29.6(17.5)$ & $31.7(13.2-40-5)$ & $20.5(16.1)$ & $13.0(7.1-35.4)$ \\
\hline Low olive oil intake & $9.2(4.5)$ & $8.0(6.3-12.8)$ & $10.4(4.6)$ & $12.3(6.9-13.6)$ & $8.7(4.4)$ & $7.7(6.2-11.6)$ \\
\hline High olive oil intake & $40.4(9.2)$ & $38.1(34.0-48.5)$ & $41.4(10.3)$ & $39.6(34.0-48.8)$ & $39.1(7.7)$ & $37.8(35.0-48.3)$ \\
\hline \multicolumn{7}{|c|}{ Plasma OA concentration $\mathrm{ng} / \mathrm{ml}$} \\
\hline Non-consumers of olive oil & $0.72(0.82)$ & $0.43(0.15-0.92)$ & $0.67(0.72)$ & $0.39(0.15-0.85)$ & $0.78(0.93)$ & $0.53(0.15-1.09)$ \\
\hline All olive oil consumers & $1.13(1.09)$ & $0.81(0.41-1.43)$ & $1.20(1.12)$ & $0.81(0.32-1.64)$ & $1.08(0.99)$ & $0.77(0.44-1.37)$ \\
\hline Low olive oil intake & $0.95(1.03)$ & $0.59(0.15-1.21)$ & $0.99(1.34)$ & $0.42(0.15-1.06)$ & $0.93(0.87)$ & $0.67(0.30-1.21)$ \\
\hline High olive oil intake & $1.32(1.14)$ & $0.96(0.46-1.91)$ & $1.33(1.15)$ & $0.98(0.47-1.91)$ & $1.31(1.14)$ & $0.94(0.46-1.73)$ \\
\hline
\end{tabular}


Difference in mean OA concentration between olive oil groups ${ }^{1}$

$\begin{array}{llll}\text { Non-consumers vs all consumers } & p=0.012 & p=0.054 & p=0.110 \\ \text { Non-consumers vs low consumers } & p=0.213 & p=0.789 & p=0.261 \\ \text { Non-consumers vs high consumers } & p=0.002 & p=0.009 & p=0.068 \\ \text { Low consumers vs high consumers } & p=0.052 & p=0.081 & p=0.310\end{array}$

Abbreviations: OA - Oleanolic acid; SD - standard deviation. ${ }^{1}$ Wilxoxon Rank-sum test 
Table 3. Correlation coefficients between olive oil intake from dietary questionnaire and plasma oleanolic acid concentration

Olive oil consumers

\begin{tabular}{lrrrrrrr} 
Correlation coefficient & \multicolumn{2}{c}{ All $(\mathrm{n}=96)$} & Men $(\mathrm{n}=42)$ & \multicolumn{2}{c}{ Women $(\mathrm{n}=54)$} \\
model & $\mathrm{r}$ & $\mathrm{p}$-value & $\mathrm{r}$ & $\mathrm{p}$-value & $\mathrm{r}$ & $\mathrm{p}$-value \\
& & & & & & \\
\hline Crude & 0.18 & 0.0724 & 0.12 & 0.441 & 0.23 & 0.090 \\
Partially adjusted $^{1}$ & 0.37 & $<0.001$ & 0.34 & 0.040 & 0.39 & 0.006 \\
Fully adjusted $^{2}$ & 0.36 & $<0.001$ & 0.33 & 0.06 & 0.37 & 0.010
\end{tabular}

${ }^{1}$ Partial Spearman correlation adjusted by centre, age and sex

${ }^{2}$ Partial Spearman correlation adjusted by centre, age, sex, total fruit intake, red wine

intake and fasting state 


\section{Graphical abstracts}

Oleanolic acid (OA) is an important minor compound in olive oil with potentially beneficial health effects. Our study determined concentrations of OA in blood in the general population for the first time and found a low-to-moderate link between blood OA and olive oil intake. Whether OA concentrations in blood are a good marker of olive oil consumed needs to be further clarified.

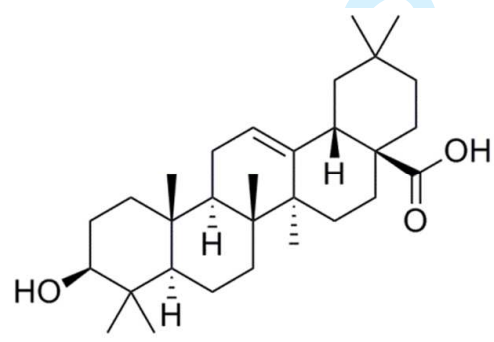

Chemical structure of Oleanolic Acid 